\title{
OPEN TRANSFORMATIONS AND DIMENSION ${ }^{1}$
}

\author{
J. H. ROBERTS
}

This paper considers separable metric spaces $A$ and $B$ and open transformations. If, for each $x \in A, f(x) \in B$ and the image under $f$ of every open set in $A$ is a set open in $B$, then $f$ is an open transformation. Continuity of $f$ is not assumed. Such transformations have been studied by Rhoda Manning [1]. ${ }^{2}$

THEOREM 1. If $f(A)=B$ where $f$ is open, then there exists a subset $A_{1}$ of $A$ such that (1) $f\left(A_{1}\right)=B$, (2) for $y \in B$, the set $f^{-1}(y) \cdot A_{1}$ is countable, and (3) $f$, considered as a transformation of $A_{1}$ into $B$, is open.

Proof. Let $K_{1}, K_{2}, \cdots$ denote the elements of a countable base (open sets) for the space $A$. For every $y \in B$ and each $i$ let $P_{y i}$ be a point of $K_{i} \cdot f^{-1}(y)$, provided this set is nonvacuous. Let $A_{1}$ be the set of all points $P_{y i}$ so obtained. Properties (1) and (2) are obvious. To prove (3), let $V$ be an open set in $A_{1}$, and $U$ an open set in $A$ such that $U \cdot A_{1}=V$. Now for every $y$ the set $f^{-1}(y) \cdot A_{1}$ is dense in $f^{-1}(y)$. Hence if $f^{-1}(y)$ has a point in $U$ then it has a point in $A_{1} \cdot U$ so $f(V)=f(U)$ is an open set in $B$.

THEOREM 2. There exist countable-fold open mappings ${ }^{3}$ which increase dimension.

Proof. There exist open mappings which increase dimension [2]. Theorem 2 follows by applying Theorem 1 to any such example. ${ }^{4}$

Theorem 3. If $\operatorname{dim} A=n$ and $-1<m \leqq n$, then there exists $a B$ and a transformation $f$ such that (1) $f(A)=B$, (2) $f$ is open and 1-1, and (3) $\operatorname{dim} B=m$. In other words, dimension can be lowered at will by a 1-1 open transformation. ${ }^{5}$

Presented to the Society, April 27, 1946; received by the editors October 14, 1946.

${ }^{1}$ One statement in the abstract (Bull. Amer. Math. Soc. Abstract 59-5-210) is incorrect. Theorem 4 gives the correct statement.

${ }^{2}$ Numbers in brackets refer to the bibliography.

${ }^{3} \mathrm{~A}$ mapping is a continuous transformation.

${ }^{4}$ Alexandroff [5] has proved that if $A$ is compact then no countable-fold open mapping can increase dimension.

5 Compare the following special case of a theorem of Hurewicz [4, p. 91, Theorem VI 7]): "If $f$ is a closed mapping of $A$ into $B$ and for each $y \in B, f^{-1}(y)$ is zero-dimensional, then $\operatorname{dim} B \geqq \operatorname{dim} A$." In a footnote (loc. cit.) the authors state that it is not known if Theorem VI 7 is true for open mappings. The answer is in the negative and their example VI 10 is a counter example, as the mapping $f$ is actually open. 
Proof. It is sufficient to prove the theorem for $m=n-1$. For if $f(A)=B$ and $g(B)=C$, where $f$ and $g$ are open and 1-1, then the product $g f$ is open and 1-1 and transforms $A$ onto $C$.

The author has shown ${ }^{6}$ that there exists a space $B_{1}$ of dimension $n-1$, and an at most 2-to-1 mapping $\phi$ of $B_{1}$ onto $A$. Furthermore the subset of $B_{1}$ consisting of all $x$ such that $\phi^{-1} \phi(x)=x$ is $(n-1)$ dimensional. For $y \in A$, if $\phi^{-1}(y)$ is a single point then write $f(y)$ $=\phi^{-1}(y)$. If $\phi^{-1}(y)$ is double-valued, select arbitrarily one point in $\phi^{-1}(y)$ and define $f(y)$ to be this point. Let $f(A)=B$. Then $B$ is of dimension $n-1$ and $f$, as inverse of a 1-1 continuous function, is open.

Remark. If $f(A)=B$ is open and $1-1$, then $f^{-1}$ is continuous and 1-1. Thus Theorem 3 provides examples of arbitrary increases of dimension by 1-1 mappings.

THEOREM 4. Suppose $f(A)=B$ is open, $B$ is locally compact, and for each $y \in B$ the set $f^{-1}(y)$ is not dense-in-itself. Then $\operatorname{dim} B \leqq \operatorname{dim} A .^{7}$

Proof. Let $K_{1}, K_{2}, \cdots$ be an open base for $A$. For each $i$ let $A_{i}$ be the set of all $x \in K_{i}$ such that if $x^{\prime} \in K_{i}$ and $f\left(x^{\prime}\right)=f(x)$, then $x^{\prime}=x$, and let $B_{i}=f\left(A_{i}\right)$. For $y \in B, f^{-1}(y)$ contains an isolated point (with respect to $\left.f^{-1}(y)\right)$, so $B=\sum_{i=1}^{\infty} B_{i}$. We prove next that $B_{i}$ is closed in the open set $f\left(K_{i}\right)$. For suppose on the contrary that there exists a sequence $y_{n} \rightarrow y$, where $y$ and each $y_{n}$ are in $f\left(K_{i}\right), y_{n} \in B_{i}$ but $y \notin B_{i}$. Since $y \in f\left(K_{i}\right)$ but not in $B_{i}$, there exist distinct points $x$ and $x^{\prime}$ in $K_{i}$ such that $f(x)=f\left(x^{\prime}\right)=y$. For each $n$ there is a unique $x_{n} \in K_{i}$ such that $f\left(x_{n}\right)=y_{n}$. Since $y_{n} \rightarrow y$ it follows that $\lim$ inf $f^{-1}\left(y_{n}\right) \supset f^{-1}(y)$ $\supset\left(x+x^{\prime}\right)$. But $f^{-1}\left(y_{n}\right)$ has only the one point $x_{n}$ in $K$. This gives a contradiction.

Now let $M_{i 1}, M_{i 2}, \cdots$ be closed and compact subsets of $B$ with $M_{i 1}+M_{i 2}+\cdots=f\left(K_{i}\right)$. Write $B_{i j}=B_{i} \cdot M_{i j}$. Then $B_{i j}$ is a compact space and $B=\sum_{i=1}^{\infty} \sum_{j=1}^{\infty} B_{i j}$. Hence if $\operatorname{dim} B_{i j} \leqq \operatorname{dim} A$ for all $i$ and $j$, then $\operatorname{dim} B \leqq \operatorname{dim} A$ (see [4, Theorem III 2, p. 30]). Write $A_{i j}$ $=A_{i} \cdot f^{-1}\left(B_{i j}\right)$. Over $A_{i j}, f$ is open and 1-1 to $B_{i j}$. For $y \in B_{i j}$ define $g(y)=x \in A_{i j}$ such that $f(x)=y$. Now $g$, as the inverse of any open 1-1 transformation, is continuous; thus $g$ is a mapping and $g\left(B_{i j}\right)=A_{i j}$. Furthermore $B_{i j}$ is compact, so that $g$ is a closed mapping. Also $g$ is 1-1. Then ${ }^{8} \operatorname{dim} B_{i j} \leqq \operatorname{dim} A$, so $\operatorname{dim} B \leqq \operatorname{dim} A$.

Remark. In Theorem 4 the assumption that $B$ is locally compact

${ }^{6}$ See [3], especially Theorem 9.1. If the $M$ of this theorem is the space $A$, then $M_{1}$ is the desired space $B_{1}$ and $\phi_{1}$ is the desired mapping $\phi$.

7 See footnote 4 .

8 See [4, Theorem VI 7, p. 91]. 
can be replaced by the weaker assumption that every point of $B$ has arbitrarily small neighborhoods with compact boundary. The proof is not given. Some assumption of compactness seems necessary. Consider the following example.

Example. There exist, in the plane, spaces $A$ and $B$ and an open 1-1 transformation $f$ with $f(A)=B$, with $\operatorname{dim} A=0$ and $\operatorname{dim} B=1$.

The space $B$ is an example due to Sierpiński [6, pp. 81-83]. This space $B$ has the following properties: (1) it is 1-dimensional, (2) it lies in the plane with $0 \leqq x \leqq 1,0 \leqq y \leqq 1,(3)$ it contains at most one point $(x, y)$ for a given $x$, and (4) the set of all points $(x, 0)$, such that for some $y$ the point $(x, y) \in B$, is homeomorphic to the Cantor ternary set. Let $A$ be this set defined by (4). Then $A$ is the projection of $B$ onto the $x$-axis, and for $(x, 0) \in A$ there is a single point $(x, y)$ in $B$. Define $f$ as follows: $f(x, 0)=f(x, y) \in B$. Then $f$ has the required properties.

\section{BIBLIOGRAPHY}

1. Rhoda Manning, Open and closed transformations, Duke Math. J. vol. 13 (1946) pp. 179-184. 38.

2. A. Kolmogoroff, Über offene Abbildungen, Ann. of Math. vol. 38 (1937) pp. 36-

3. J. H. Roberts, A theorem on dimension, Duke Math. J. vol. 8 (1941) pp. 565-574.

4. W. Hurewicz and H. Wallman, Dimension theory, Princeton University Press.

5. P. Alexandroff, Über abzählbar-facheoffene Abbildungen, C. R. (Doklady) Acad. Sci. URSS. vol. 4 (1936) pp. 295-299.

6. W. Sierpiński, Sur les ensembles connexes et non connexes, Fund. Math. vol. 2 (1921) pp. 81-95.

Duke University 